\title{
Dual role for macrophages in vivo in pathogenesis and control of murine Salmonella enterica var. Typhimurium infections
}

\author{
Odilia L. C. Wijburg ${ }^{1}$, Cameron P. Simmons ${ }^{1,2}$, Nico van Rooijen ${ }^{3}$ and Richard A. \\ Strugnell ${ }^{1,2}$ \\ ${ }^{1}$ Department of Microbiology and Immunology, The University of Melbourne, Melbourne, \\ Australia \\ ${ }^{2}$ CRC for Vaccine Technology, The University of Melbourne, Melbourne, Australia \\ ${ }^{3}$ Department of Cell Biology and Immunology, Vrije Universiteit, Amsterdam, The Netherlands
}

Salmonella spp. are regarded as facultative intracellular bacterial pathogens which are found inside macrophages (M $\Phi)$ after i. v. infection. It is generally assumed that $M \Phi$ restrict the replication of the bacteria during infection. In this study we examined the in vivo activities of $\mathrm{M} \Phi$ during experimental S. typhimurium infections, using a selective liposome-based M $\Phi$ elimination technique. Unexpectedly, elimination of $M \Phi$ prior to infection with virulent S. typhimurium decreased morbidity and mortality, suggesting that $\mathrm{M} \Phi$ mediate the pathology caused by S. typhimurium. Removal of $M \Phi$ during vaccination with attenuated S. typhimurium did not affect protection against challenge with virulent $S$. typhimurium, suggesting that $\mathrm{M} \Phi$ are not required for the induction of protective immunity and that other cells must function as antigen-presenting cell to elicit T cell-mediated protection. However, $M \Phi$ appeared to be important effectors of protection against challenge infection since elimination of $M \Phi$ from vaccinated mice prior to challenge infection with virulent S.typhimurium significantly decreased protection. These results enhance our understanding of the control of S. typhimurium growth in vivo, and moreover suggest that $\mathrm{M} \Phi$ play a major role in the pathology of virulent S. typhimurium infections. As such, these cells may present a novel target for therapeutic intervention.

Key words: Salmonella enterica / Pathogenesis / Innate resistance / Macrophage / Enteric infection

\begin{tabular}{|lr|}
\hline Received & $23 / 6 / 99$ \\
Revised & $13 / 12 / 99$ \\
Accepted & $14 / 12 / 99$ \\
\hline
\end{tabular}

\section{Introduction}

Salmonella enterica are significant worldwide pathogens which cause localized and systemic diseases in many animal species. The World Health Organisation estimates that typhoid fever, the most important human salmonellosis, is responsible for 600000 deaths from 16 million cases worldwide [1]. S. enterica var. typhimurium cause a systemic disease in mice which has been used to model the pathogenesis and immunology of human typhoid fever. Studies of oral S. enterica infections in humans and mice suggest that Salmonella spp. are facultative intracellular bacterial pathogens which invade

[l 19797]

Abbreviations: $\mathrm{CL}_{2}$ MDP: Dichloromethylene diphosphonate C-frag: C-fragment of tetanus toxin DC: Dendritic cell(s) RES: Reticuloendothelial system NO: Nitric oxide the gastrointestinal mucosa through the specialized M cells in the Peyer's patches, then spread into the draining mesenteric lymph nodes where they are thought to reside and multiply in $\mathrm{M} \Phi[2,3]$. From the mesenteric lymph nodes, bacteria disseminate via the thoracic duct into the bloodstream, and are removed by the $M \Phi$ of the reticuloendothelial system (RES), mainly in the spleen, liver and bone marrow [2, 3]. Dogma states that Salmonella multiply in the RES M $\Phi$ and are seeded back into the bloodstream, possibly following apoptosis of these cells, until a T cell-mediated immune response begins to control infection through up-regulation of RES MФ. In the absence of a $T$ cell response, the infection is fatal to mice $[4,5]$.

This murine salmonellosis model holds that the $M \Phi-$ Salmonella interaction is central to the early control of S. typhimurium growth, and the subsequent induction of acquired immunity $[6,7]$. Much of the evidence used to support this hypothesis comes from in vitro studies in

(C) WILEY-VCH Verlag GmbH, D-69451 Weinheim, 2000 
which $M \Phi$-like cell lines or bone marrow-derived $M \Phi$ were used [8-11]. These in vitro studies showed that S. typhimurium is able to survive and replicate in phagosomes of non-activated $\mathrm{M} \Phi$ and, more recently, that this replication can lead to apoptosis [11]. In contrast, in vivo studies have suggested that $S$. typhimurium may reside inside multiple cells types including neutrophils, $M \Phi$ and/or hepatocytes; only one recent study has shown the exclusive localization of S.typhimurium in $M \Phi$ [12-14].

A role for the $M \Phi$ in innate, genetically determined resistance to infection [15], and subsequent acquired resistance to reinfection, was inferred from gene mapping studies which demonstrated that natural resistance is mediated by a locus on chromosome 1 called Nramp1, which coincidently confers resistance to diseases caused by other $\mathrm{M} \Phi$-associated pathogens, e. g. Mycobacterium [16]. The natural resistance-associated $M \Phi$ protein (Nramp1) is almost exclusively expressed by $M \Phi$ and susceptibility and resistance to murine S. typhimurium infection is determined by a mutation in Nramp1 which results in a single amino acid substitution $[16,17]$. Mice expressing the resistant allele of Nramp1 can control the growth rate of $S$. typhimurium in vivo, allowing the development of acquired immune responses.

The induction of acquired, predominantly $T$ cellmediated, immune responses is essential to the eventual clearance of S. typhimurium and recovery from infection $[4,5,18]$. Salmonella-derived peptides are efficiently presented by $M \Phi$ to $T$ cells in vitro [19, 20], suggesting that $M \Phi$ might function as APC in vivo for the induction of protective $\mathrm{T}$ cell responses. However, most of these in vitro studies used T cell hybridoma proliferation to quantitate antigen presentation by $M \Phi$ or $M \Phi$-like cell lines. It is now recognized that while $T$ cell hybridomas can be stimulated by $M \Phi$ in vitro, dendritic cells $(D C)$ are the principal APC involved in activation of naive T cells in vivo [21]. A primary role for $M \Phi$ as APC for the induction of acquired immune responses against $S$. typhimurium in vivo therefore remains unproven.

In this study we examined the role of $\mathrm{M} \Phi$ in vivo in protection and induction of acquired immune responses against experimental S. typhimurium infections. We used a liposome-based $M \Phi$ depletion technique [22] to eliminate $M \Phi$ in vivo. This technique has been studied extensively and is based on the liposome-mediated intracellular delivery of the drug dichloromethylene diphosphonate $\left(\mathrm{Cl}_{2} \mathrm{MDP}\right)$. Accumulation of $\mathrm{Cl}_{2} \mathrm{MDP}$ in the cytosol disturbs the metabolism of the $M \Phi$, leading to irreversible damage, ultimately resulting in their death and depletion from the tissues in the absence of an inflammatory response [22]. The selective depletion of $M \Phi$ has been confirmed in a large number of in vivo studies, both ultrastructurally and by loss of $M \Phi$-specific markers [22]. Several studies have shown that the $\mathrm{Cl}_{2} \mathrm{MDP}$-liposomes do neither affect the tissue architecture nor the presence and function of other phagocytic cells, such as blood monocytes, granulocytes or DC [23-25].

In the present study we compared Salmonella infections in $M \Phi$-depleted mice and normal mice, and our results contribute to the current understanding of the importance of the in vivo $M \Phi-S$. typhimurium interaction in the initial control of the infection, and for vaccine-mediated protection against Salmonella infections. Our studies suggest that $M \Phi$ play a dual role in S. typhimurium infections. We were able to show that $M \Phi$ are centrally involved in the morbidity and mortality caused by virulent S. typhimurium infections in naive animals, and as immune effectors which facilitate clearance of bacteria and recovery from infection in vaccinated mice.

\section{Results}

\subsection{In vivo elimination of $M \Phi$ using a liposome- mediated depletion technique}

In this study, we used a liposome-mediated M $\Phi$ depletion technique to eliminate spleen and liver $M \Phi$ in vivo. Mice were injected i.v. with $0.1 \mathrm{ml} \mathrm{Cl}$ MDP-liposomes per $10 \mathrm{~g}$ body weight, either 2 days before immunization and/or 2 days before challenge with virulent S. typhimurium. This treatment resulted in the complete elimination of $M \Phi$ residing in the red pulp and marginal zone area of the spleen (Fig. 1) and in the liver (results not shown).

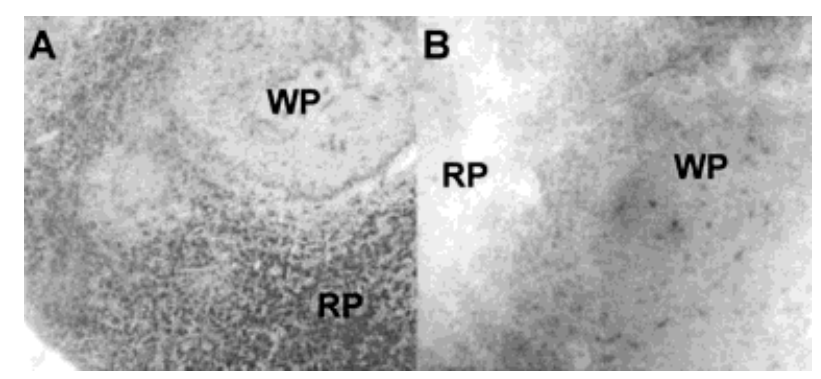

Fig. 1. Effect of $\mathrm{Cl}_{2} \mathrm{MDP}$-liposome administration on $\mathrm{M} \Phi$ populations in the spleen. Shown are cryostat sections of spleens obtained from normal $(\mathrm{A})$ and $\mathrm{Cl}_{2} \mathrm{MDP}$-liposometreated (B) mice stained for acid phosphatase. The $\mathrm{Cl}_{2} \mathrm{MDP}$ liposomes were administered i.v. 2 days earlier, resulting in complete elimination of red pulp $\mathrm{M} \Phi$ and marginal zone $\mathrm{M} \Phi$. $\mathrm{RP}=$ red pulp, WP $=$ white pulp. 


\subsection{Effect of in vivo MФ depletion on challenge with virulent $S$. typhimurium}

To determine the role of $\mathrm{M} \Phi$ in controlling low dose wildtype infection by virulent S. typhimurium SL1344, normal and $M \Phi$-depleted mice were infected i.v. with $100 \mathrm{CFU}$ SL1344, and monitored for survival against time (Fig. 2). All normal mice and mice treated with PBS-liposomes 2 days before infection died within 6-7 days after infection, whereas mice treated once with $\mathrm{Cl}_{2} \mathrm{MDP}$-liposomes survived the infection for a longer period, and died 8-9 days after infection. These results suggested that $M \Phi-$ depleted naive mice were more resistant to mortality as a result of $S$. typhimurium infection in comparison with normal mice. To maintain the depletion of $M \Phi$ from the tissues [26], mice were given $50 \mu \mathrm{l} \quad \mathrm{Cl}_{2} \mathrm{MDP}$ liposomes on day 3 and day 8 after infection. Mice which were therefore continuously depleted of $M \Phi$ [26] survived up to 10 days after infection (Fig. 2).

To further study the effect of $M \Phi$ depletion on protection of mice against infection with virulent S. typhimurium SL1344, the number of bacteria in spleens and livers of infected animals was determined at indicated time points after i.v. inoculation with 100 CFU SL1344. Macroscopic examination of SL1344-infected mice showed a significant enlargement of spleen (Fig. $3 \mathrm{~A}$ ) and liver (hepatosplenomegaly) and widespread necrotic lesions in the organs in normal and PBS-liposome-treated mice on day 3 and 4 which worsened in time, whereas the organs of $\mathrm{Cl}_{2} \mathrm{MDP}$-liposome-treated mice showed no signs of pathology until day 6 after infection (results not shown). In untreated infected mice, bacterial counts increased approximately tenfold per day, and reached to approximately $2.2 \times 10^{8}$ in the spleen (Fig. 3 B) and $2.6 \times$

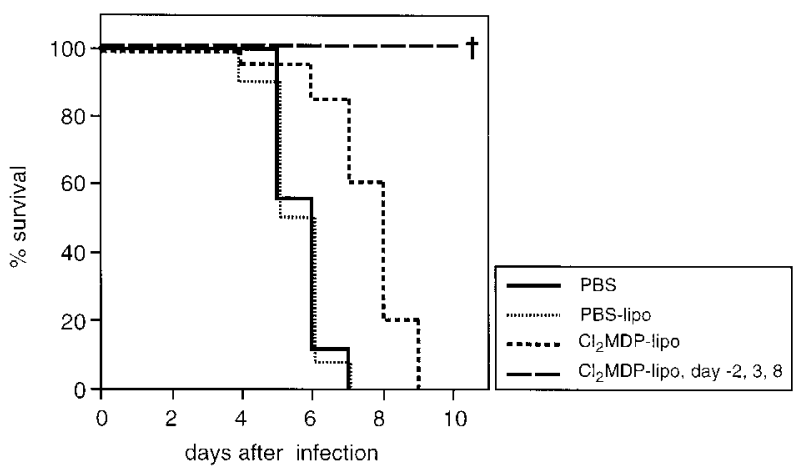

Fig. 2. $M \Phi$ depletion increases resistance to virulent S. typhimurium infection. Groups of five mice were injected i. v. with either PBS, PBS-liposomes or $\mathrm{Cl}_{2} \mathrm{MDP}$-liposomes 2 days before i.v. infection with $10^{2}-10^{2.3} \mathrm{CFU}$ S. typhimurium strains SL1344. One group of mice received $\mathrm{Cl}_{2} \mathrm{MDP}$ liposomes every 5 days to maintain $M \Phi$ depletion. The results are presented as the cumulative survival of mice in three independently performed experiments. + Mice in this group became moribund and the experiment was stopped.

$10^{8}$ in the liver (Fig. 3 C) 5 days after infection. The number of bacteria in mice treated with PBS-liposomes was equivalent to those found in untreated infected mice (results not shown). Treatment of mice with $\mathrm{Cl}_{2} \mathrm{MDP}$ liposomes 2 days before infection with SL1344 resulted in a reduction of bacterial load in both the spleen and the liver. A significant $(p=0.001) 10$ - to 50 -fold reduction in the number of SL1344 was observed in the spleen on day 4 after infection, and on day 5 the bacterial load was 100-fold lower compared with untreated infected mice. Similarly, treatment with $\mathrm{Cl}_{2} \mathrm{MDP}$-liposomes before inoculation of SL1344 resulted in a reduced bacterial load in

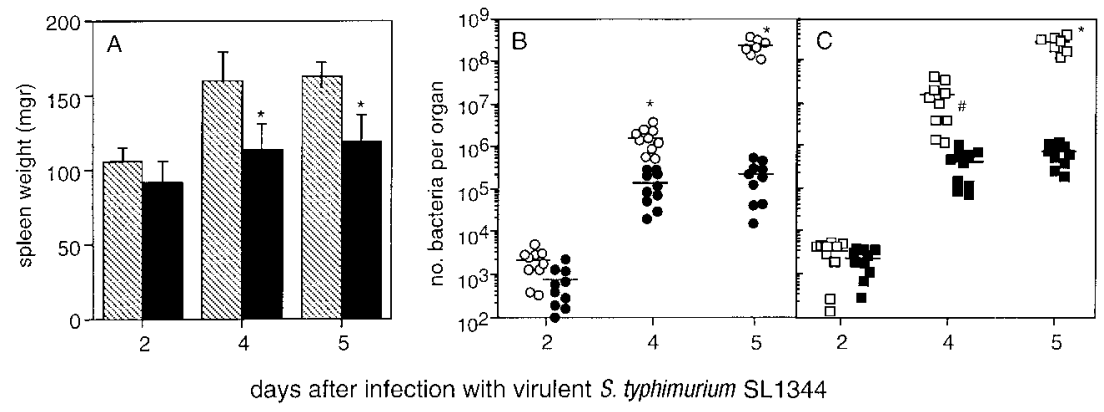

Fig. 3. Replication of virulent S. typhimurium is inhibited in M $\Phi$-depleted mice. Groups of ten mice were treated i.v. with PBS (open symbols) or $\mathrm{Cl}_{2} \mathrm{MDP}$-liposomes (closed symbols) 2 days before i. v. infection with $100 \mathrm{CFU}$ S. typhimurium SL1344, and received $\mathrm{Cl}_{2}$ MDP-liposomes on day 3 to maintain the $\mathrm{M} \Phi$ depletion. Shown are spleen weights (A, mean $\pm S E$ ) of PBS (שי )- and $\mathrm{Cl}_{2}$ MDP-liposome treated mice $(\square)$, and the number of bacteria in spleen (B) and liver homogenates $(C)$ as determined by viable counts on LB-media. * and \# Indicate a statistically significant difference ( ${ }^{*} p<0.01, \# p<0.05$ ) between the PBS- and Cl ${ }_{2} \mathrm{MDP}$ liposome-treated mice at indicated time points in a Mann-Whitney U-test. The presented results are one representative out of six independently performed experiments. 
the liver (Fig. $3 \mathrm{C}$ ). These experiments were performed at least six times, and in each experiment the in vivo growth rate of SL1344 was equivalent, and the level of growth of the bacteria in normal (non-depleted) mice was always much greater than the bacterial growth rate in $\mathrm{M} \Phi$ depleted mice. These results suggested that in vivo replication of S. typhimurium was reduced in the absence of MФ. The growth rates of SL1344 and BRD509 incubated in vitro with up to $1 \mathrm{mg} / \mathrm{ml} \mathrm{Cl} \mathrm{Cl}_{2} \mathrm{MDP}$ was not affected (data not shown).

\subsection{Effect of in vivo МФ depletion on induction of acquired immune responses}

To study the importance of in vivo $\mathrm{M} \Phi-S$. typhimurium interactions for the induction of acquired immune responses against expressed by S.typhimurium, we compared the generation of antigen-specific antibody responses in normal and $\mathrm{M} \Phi$-depleted mice after i.v. immunization with an attenuated, aromatic-dependent mutant of S. typhimurium expressing the C-fragment of tetanus toxin (C-frag). $\mathrm{Cl}_{2} \mathrm{MDP}$-liposomes were injected 2 days before immunization and every 5 days thereafter to maintain $\mathrm{M} \Phi$ depletion throughout the experiment. Treatment of mice with $\mathrm{Cl}_{2} \mathrm{MDP}$-liposomes prior to and during immunization with BRD509/C-frag neither altered the number of bacteria in the spleen or liver nor the bacterial clearance kinetics, and did not cause mortality among the vaccinated mice (Fig. 4). In addition, treatment of normal and $\mathrm{M} \Phi$-depleted mice with gentamicin during immunization with BRD509/C-frag did not affect bacterial load in the organs, suggesting that all recov-

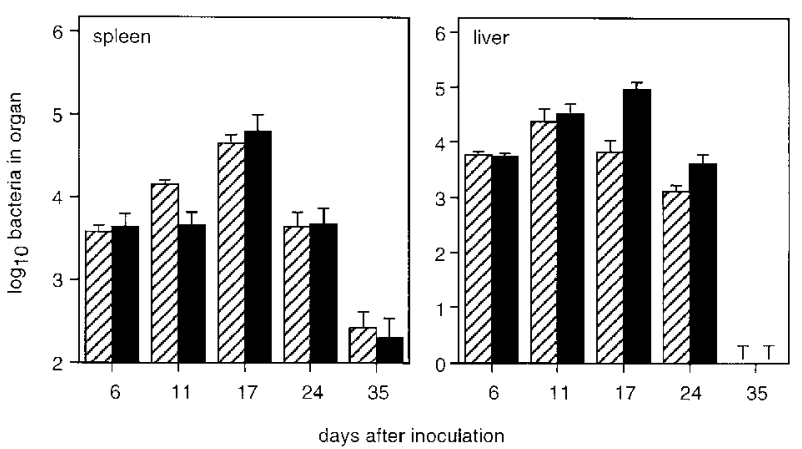

Fig. 4. М $\Phi$ depletion does not affect growth or clearance of attenuated S.typhimurium. PBS-treated mice $(\square)$ and $\mathrm{Cl}_{2}$ MDP-liposome-treated mice $(\square)$ were inoculated i.v. with $10^{5} \mathrm{CFU}$ BRD509/C-frag. M $\Phi$ depletion was maintained throughout the experiment by injecting $\mathrm{Cl}_{2}$ MDP-liposomes i.v. every 5 days. The number of bacteria in spleen and liver homogenates was determined by viable counts on LB media. Presented are the mean \pm SE of groups of five mice. ered bacteria were residing inside cells (results not shown).

Immunized mice were bled weekly and serum antibodies against $\mathrm{C}$-frag or $\mathrm{S}$. typhimurium LPS were measured by ELISA. Fig. 5 shows that $\mathrm{Cl}_{2} \mathrm{MDP}$-liposome treatment before and during immunization with BRD509/C-frag did not affect the induction of antigen-specific antibodies, since both the kinetics of the response, and serum end point titers were equivalent in untreated and $\mathrm{Cl}_{2} \mathrm{MDP}$ liposome-treated mice. Moreover, both groups of mice showed similar isotype distribution in the LPS- and TTspecific humoral responses (results not shown).

To further determine the effect of the absence of $M \Phi$ on the induction of acquired immune responses, antigenspecific $\mathrm{T}$ cell proliferation was measured in normal and $\mathrm{M} \Phi$-depleted mice after immunization with either BRD509 or BRD509/C-frag. Splenic T cells of immunized mice were cultured for 4 days with recombinant $\mathrm{C}$-frag. T cells obtained from naive mice or from mice immunized with BRD509 did not proliferate upon stimulation with $\mathrm{C}$-frag, whereas $\mathrm{T}$ cells isolated from BRD509/C-frag-immunized mice showed antigendependent proliferation. As is evident from Fig. 6, $\mathrm{Cl}_{2} \mathrm{MDP}$-liposome treatment before and during immunization with BRD509/C-frag resulted in significantly increased $T$ cell proliferative responses $(p<0.01)$ towards $\mathrm{C}$-frag in comparison with $\mathrm{C}$-frag-specific $\mathrm{T}$ cell responses in untreated immunized mice. Nonspecific $T$ cell proliferation induced with ConA was not affected by $\mathrm{Cl}_{2} \mathrm{MDP}$-liposome treatment (results not shown), sug-

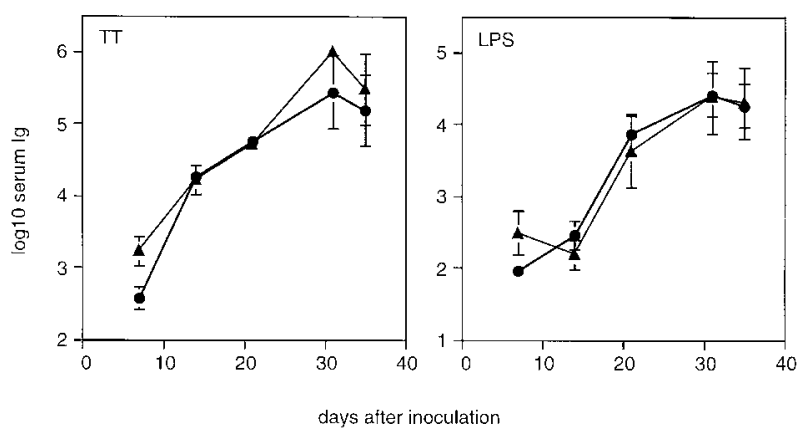

Fig. 5. $\mathrm{M} \Phi$ depletion does not alter the serological response to S.typhimurium. PBS-treated mice (O) and $\mathrm{Cl}_{2}$ MDP-liposome-treated mice $(\mathbf{\Delta})$ were immunized i. v. with $10^{5} \mathrm{CFU}$ BRD509/C-frag. Every 5 days, $\mathrm{Cl}_{2} \mathrm{MDP}$-liposomes were injected i.v. to maintain $M \Phi$ depletion throughout the experiment. Serum antibody titers specific for tetanus toxin and S. typhimurium-LPS were measured by ELISA and are presented as the $\log _{10}$ of the highest dilution with an absorbance of 0.1 above normal serum. Shown are mean \pm SE of groups of five mice. 


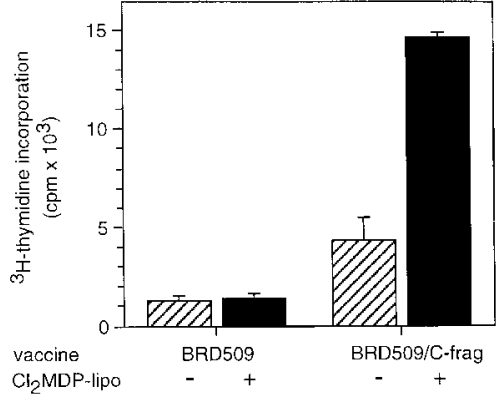

Fig. 6. $\mathrm{M} \Phi$ depletion increases $\mathrm{T}$ cell responses to S. typhimurium-associated antigens. Groups of five PBStreated mice (泪) and $\mathrm{Cl}_{2} \mathrm{MDP}$-liposome-treated mice (ם) were immunized i.v. with $10^{5}$ CFU BRD509 or BRD509/Cfrag. $\mathrm{Cl}_{2} \mathrm{MDP}$-liposomes were injected i. v. every 5 days to maintain $M \Phi$ depletion. T cell proliferation assays were performed 10 days after immunization. Shown is $\left[{ }^{3} \mathrm{H}\right]$ thymidine incorporation of pooled splenic T cells cultured with $1 \mu \mathrm{g} / \mathrm{ml}$ C-frag.

gesting that the increased C-frag-specific T cell proliferation in $\mathrm{Cl}_{2} \mathrm{MDP}$-liposome-treated mice was not due to an increased proliferative capacity of these $T$ cells.

\subsection{Effect of in vivo $\mathrm{M \Phi}$ depletion on induction of protective immunity against virulent S. typhimurium}

Finally, we examined whether in vivo $\mathrm{M} \Phi$ depletion during vaccination with BRD509 would affect induction of protective immunity against challenge with virulent S. typhimurium. Mice were depleted of $\mathrm{M} \Phi$ either during vaccination with BRD509/C-frag and/or during challenge with virulent SL1344. Protection was measured by counting the number of SL1344 bacteria in spleen and liver 4 days after challenge (Fig. 7). Normal, BRD509vaccinated mice were highly resistant to challenge with SL1344, as was evident from the $10^{5}$-fold lower number of bacteria in their tissues compared with normal, nonvaccinated mice $(p<0.01)$. Treatment of BRD509vaccinated mice with $\mathrm{Cl}_{2} \mathrm{MDP}$-liposomes during immunization did not significantly alter protection against challenge with SL1344, and the bacterial load of these mice was similar to that of normal BRD509-vaccinated mice $(p=0.2)$. In contrast, elimination of $M \Phi$ in BRD509vaccinated mice at the time of challenge with SL1344 significantly reduced protection, as evidenced by a reduced clearance of the challenge inoculum from both the liver and the spleen $(p<0.01)$. Mice treated with $\mathrm{Cl}_{2} \mathrm{MDP}$-liposomes both during vaccination with BRD509 and at challenge with SL1344 also showed reduced capacity to clear the challenge inoculum com-

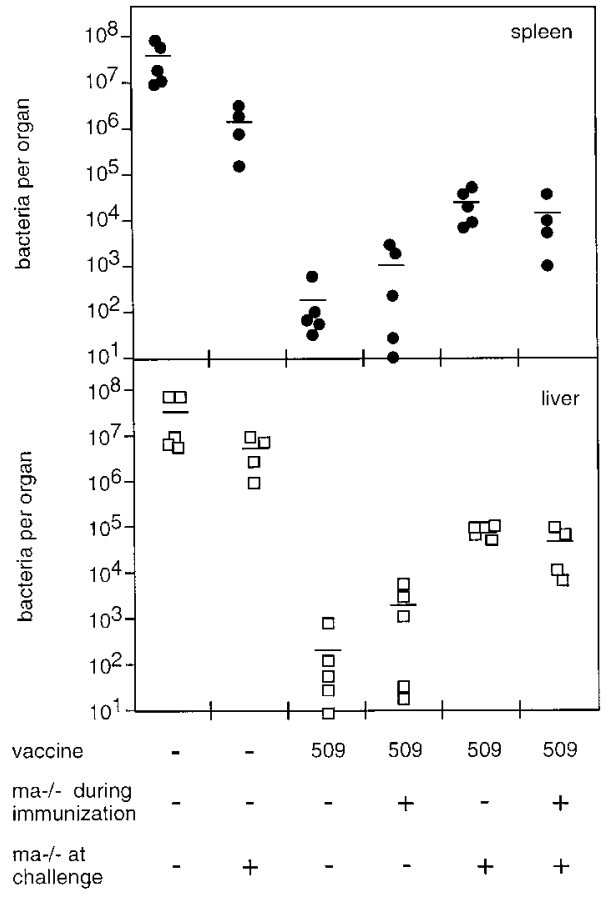

Fig. 7. Elimination of $M \Phi$ in vaccinated mice affects protection against virulent $S$. typhimurium infection. Naive and BRD509-vaccinated mice (10 $\mathrm{CFU}$ i.v.) with $100 \mathrm{CFU}$ S. typhimurium SL1344 70 days after vaccination. M $\Phi$ were eliminated (ma-/-) with $\mathrm{Cl}_{2} \mathrm{MDP}$-liposomes either before challenge or during vaccination, or both during vaccination and challenge. The number of SL1344 bacteria in tissue homogenates was determined 4 days after challenge by viable count on LB media.

pared with normal BRD509-vaccinated mice $(p<0.05)$. However, the number of SL1344 in spleen and liver of these mice was equivalent to the number of bacteria in BRD509-vaccinated mice which were depleted of $M \Phi$ at time of challenge only. These results suggested that the presence of $M \Phi$ was not essential for the induction of protective immunity during vaccination with BRD509, but that $M \Phi$ were involved in clearance of virulent S. typhimurium in vaccinated mice.

To further study the role of $M \Phi$ as effectors of protection against virulent $S$. typhimurium infection, we determined survival of mice after challenge with SL1344. Fig. 8A shows the effect of $\mathrm{Cl}_{2} \mathrm{MDP}$-liposome or PBS-liposome treatment during vaccination with BRD509 on survival of a challenge with SL1344. The vaccinated mice were able to control and survive the low-dose infection with virulent SL1344, and only one out of five normal vaccinated mice and one out of five PBS-liposome-treated vaccinated mice died, whereas all $\mathrm{Cl}_{2} \mathrm{MDP}$-liposome-treated mice lived. In contrast, injection of $\mathrm{Cl}_{2} \mathrm{MDP}$-liposomes in BRD509 vaccinated mice before challenge with SL1344 


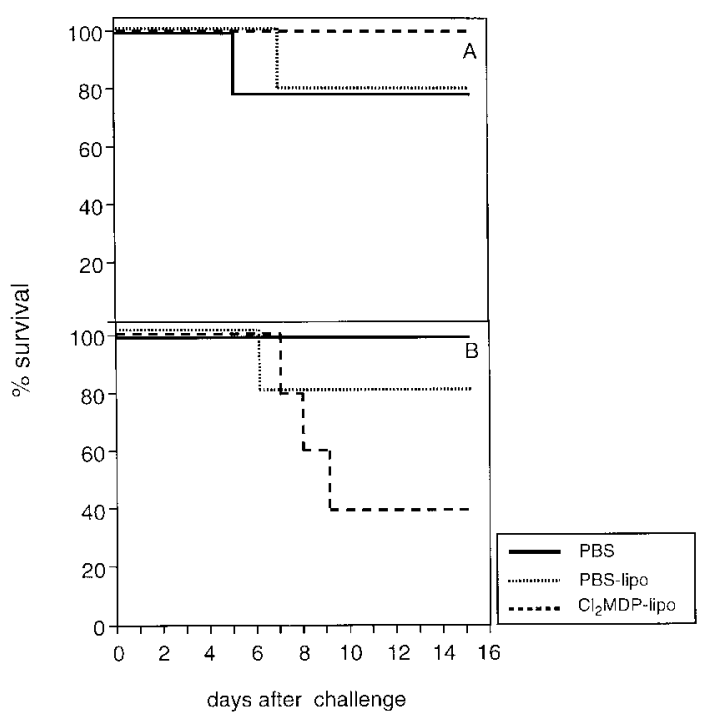

Fig. 8. Elimination of $M \Phi$ at time of challenge with virulent S. typhimurium reduces survival of immunized mice. Groups of five mice were vaccinated i.v. with $10^{5}$ CFU BRD509 and 70 days later challenged i. v. with $100 \mathrm{CFU}$ virulent S. typhimurium SL1344. Shown is the effect of treatment with PBS, PBS-liposomes or $\mathrm{Cl}_{2} \mathrm{MDP}$-liposomes during vaccination (A) or at the time of challenge $(B)$ of the immunized mice. Represented is the \% survival after challenge; all mice that were alive at day 16 after challenge survived.

did affect protection and significantly increased mortality compared with normal or PBS-liposome-treated mice (Fig. 8B). These results confirmed our observations that elimination of $M \Phi$ during immunization did not affect protection against virulent S.typhimurium, whereas removal of $M \Phi$ from immunized mice before challenge with virulent $S$. typhimurium significantly reduced protection.

\section{Discussion}

In this study we investigated the importance of the in vivo M $\Phi$-S. typhimurium interaction in the immunobiology of murine salmonellosis. Most of the previous studies investigating the interaction between $\mathrm{M} \Phi$ and Salmonella used $M \Phi$-like cell lines or bone marrow-derived $\mathrm{M} \Phi$ for in vitro studies under conditions which do not necessarily reflect the in vivo situation [8-11]. Here, we used a highly specific technique to eliminate $M \Phi$ in vivo, which does not affect the in vivo function or presence of any other phagocytic cells such as granulocytes or DC [22-25], and obtained relevant information regarding the involvement of $M \Phi$ in murine defense against S. typhimurium infection.
Several early reports pointed to a role for $M \Phi$ in natural resistance to Salmonella infections. Hormaeche [7] used radiation chimeras and showed that early resistance was mediated by bone marrow-derived, radiation-resistant cells, most likely $М \Phi$. In other reports it was demonstrated that innate resistance to S.typhimurium was decreased in silica-treated mice, presumably due to the destruction of $M \Phi$ in these mice [27]. In this study we showed that in naive mice, in vivo elimination of $M \Phi$ before and during infection with virulent $S$. typhimurium (SL1344) resulted in significantly reduced bacterial loads in spleen and liver for at least 6 days after infection, suggesting that during the initial stages of infection, S. typhimurium may reside in several cell types but replicates inside $M \Phi$. Notably, bacterial numbers in $M \Phi$-depleted mice increased from day 6 after infection despite the continuous depletion of $M \Phi$, suggesting that in the absence of $M \Phi$, S. typhimurium is able to find an alternative niche for replication.

The cellular location of S. typhimurium in vivo has been studied by several groups, and evidence has been presented that the bacteria may reside and/or replicate in $M \Phi$, neutrophils and/or hepatocytes [12-14]. In a recent in vivo study using confocal microscopy, RichterDahlfors et al. showed that $S$. typhimurium resides in $M \Phi$ in the liver [14]. In addition, Gulig et al. showed that $M \Phi$ serve as the primary host cell for Spv-mediated intracelIular replication [28]. Our results confirm the previous findings and provide further evidence that virulent S. typhimurium replicates in $M \Phi$ in vivo.

In this study we showed that elimination of $M \Phi$ before infection with virulent S.typhimurium delayed the appearance of necrotic foci in the liver and the onset of disease, and extended the mean survival time. In addition, the absence of hepatosplenomegaly in $M \Phi-$ depleted mice until the time point at which S. typhimurium replication increased (i.e. day 6 after infection) indicated an impaired recruitment of inflammatory cells in $M \Phi$-depleted animals (data not shown). Taken together, these results suggest that $M \Phi$ not only provide a niche for virulent Salmonella to grow, but are also centrally involved in the pathogenesis of murine salmonellosis. Clinical symptoms of human S. typhi infection include enteric fever and organ failure as a result of extensive damage to these organs, reportedly due to the host inflammatory response triggered by LPS. Recently, Khan et al. [29] constructed a Salmonella mutant defective in secondary acylation of the lipid A domain of the LPS molecule, reducing its ability to induce cytokines and inducible nitric oxide synthase in $M \Phi$. When injected into normal mice, the LPS-mutant Salmonella replicated to very high levels in the liver and spleen, but most of the animals survived and cleared the infection, suggesting 
that mortality after infection with S.typhimurium is dependent on the toxicity of LPS. Collectively, these and our findings, and the previously reported fact that LPSinduced cytokine secretion and LPS-related biological responses are reduced in $M \Phi$-depleted mice [30], make it tempting to speculate that, during the early stages of S. typhimurium infection, bacterial (cell wall-associated) LPS stimulates the production of inflammatory mediators from $M \Phi$ resulting in pathology and onset of disease. On the other hand, the $M \Phi$ inflammatory response is crucial to control S. typhimurium infections, since the $M \Phi$-depleted mice were not able to control the infection. Indeed, it has been shown that mice which have a genetic defect in the Ips gene and which are therefore hyporesponsive to the biological effects of LPS, are not able to control S. typhimurium infections [31]. Certainly, additional experiments involving the measurement of inflammatory cytokines during S. typhimurium infection in $\mathrm{M} \Phi$-depleted mice are warranted to provide more insight into how the M $\Phi$-Salmonella interaction initiates pathogenesis.

In contrast to our observations with virulent Salmonella, elimination of $\mathrm{M} \Phi$ before and during immunization with the attenuated $\triangle$ aroA $\triangle a r o D$ S. typhimurium (i.e. BRD509) neither altered the bacterial load in spleen or liver nor the clearance kinetics of the bacteria. Treatment with gentamicin, which should kill all extracellular bacteria, did not affect the number of bacteria in either normal or $М \Phi$-depleted mice, suggesting that the bacteria were residing intracellularly in $М \Phi$-depleted animals. Recently, Gulig et al., using i.v. M $\Phi$ depletion in combination with oral infection, showed that treatment of mice with $\mathrm{Cl}_{2} \mathrm{MDP}$-liposomes did not significantly affect growth of Spv $v^{-}$. typhimurium in the spleen measured 4 days after infection [28]. Together, these results show that virulent and attenuated strains of S.typhimurium behave differently in vivo, and may reside in different cells.

It has generally been implied that $M \Phi$ function as the principal APC in vivo for the induction of protective acquired $T$ cell responses [22, 23]. However, the only suggestive evidence for this comes from recent studies on the effect of Nramp1 on antigen processing and presentation, showing that Nramp1 influences processing of antigens and up-regulates expression of MHC class II molecules, and more indirectly affects the activation of $\mathrm{CD}^{+} \mathrm{T}$ cells by inducing secretion of M $\Phi$ cytokines such as TNF- $\alpha$ and IL-1 $\beta$, which might act as co-stimulatory factors [32, 33]. In this study, we further examined the importance of the in vivo interaction between $М \Phi$ and Salmonella for the induction of vaccine-mediated, antigen-specific and protective immune responses. We found that $М \Phi$ depletion did not affect the induction of antigen-specific antibodies, and moreover, resulted in increased antigen-specific $\mathrm{T}$ cell responses. These results indicate that $M \Phi$ are not the principal APC involved in the induction of antigen-specific immune responses induced with non-virulent strains of S. typhimurium, but imply that attenuated Salmonella must reside in other cells than $\mathrm{M} \Phi$, which either act as professional APC themselves, or pass antigens on to professional APC. Since the outcome of most recent studies pointed to DC as the main APC for the initiation of primary immune responses in vivo [21] and $M \Phi$ elimination did not affect the induction of $T$ cell responses, we suggest that DC function as the main APC for the induction of Salmonella-specific $T$ cell and antibody responses. A recent report has shown that in vitro, DC can take up Salmonella [34] and in addition, Hopkins and Kraehenbuhl [35] have shown that DC in the Peyer's patches colocalize with attenuated mutants of $S$. typhimurium.

Finally, the results of our study showed that $M \Phi$ are not involved in the induction of protective immunity, since elimination of $M \Phi$ during immunization with attenuated S. typhimurium did not affect protection against a challenge with virulent S. typhimurium. Importantly, elimination of $M \Phi$ at the time of challenge did partially affect protection against virulent $S$. typhimurium, implying that $\mathrm{M} \Phi$ are an important effector cell of the host protective response. Elimination of $M \Phi$ from immunized mice resulted in an increased bacterial burden in spleen and liver 4 days after challenge, and augmented susceptibility and mortality. Depletion of $M \Phi$ both during immunization and at the time of challenge did not further affect the protection of the mice. These results are in contrast with our observations in naive mice infected with virulent S. typhimurium, where $\mathrm{M} \Phi$ elimination resulted in a lower bacterial load and increased survival, and prove that in immune mice, $М \Phi$ play a major role in early control of bacterial growth in the liver and spleen, and are the main effector cell restricting bacterial replication in immunized mice. However, immune $\mathrm{M} \Phi$-depleted mice were still more resistant (40\% survival, Figs. 7 and 8 ) to challenge infection with virulent $S$. typhimurium than naive $\mathrm{M} \Phi-$ depleted mice (0\% survival, Figs. 2 and 3), suggesting that other immune effector mechanisms help to protect immunized mice against virulent challenge infection. Results of preliminary studies in our laboratory investigating the effect of transfer of immune serum into naive normal and $\mathrm{M} \Phi$-depleted mice before infection with virulent S. typhimurium have suggested that serum factors might form an additional component mediating protection. Furthermore, our results also suggest that, although antigen-specific acquired $\mathrm{T}$ cell responses are essential for complete recovery from virulent Salmonella infections $[5,18]$, these responses by themselves are not sufficient to control Salmonella infections in $\mathrm{M} \Phi$-depleted mice. In 
immune mice, however, bactericidal activities of $M \Phi$ may be enhanced by IFN- $\gamma$ derived form activated antigen-specific $\mathrm{T}$ cells, resulting in a more rapid clearance of bacterial infections, and on the other hand, activated $\mathrm{M} \Phi$ may secrete cytokines such as IL-12 which may enhance $\mathrm{T}$ cell activation and secretion of IFN- $\gamma$. The importance of such cytokine-related interactions between $\mathrm{M} \Phi$ and immune $T$ cells was recently shown in humans which lacked the $\beta$-chain of the IL-12 receptor [36]. These individuals were deficient in signaling through the IL-12 receptor and in the production of IFN- $\gamma$, and were unable to control mycobacterial and Salmonella infections.

Taken together, our results are important for the understanding of the host response to S.typhimurium, and provide further insight into the relevance of in vivo $\mathrm{M} \Phi-$ S. typhimurium interactions for the immunobiology of murine salmonellosis. We suggest that the interaction between $M \Phi$ and $S$. typhimurium is significant for the pathology of infection and much less important in the induction of protective immunity. The experiments described here were performed in Ity $^{\mathrm{s}}$ mice, which are known to harbor a single point mutation in the Nramp1 gene, resulting in a significant reduction in innate resistance against $S$. typhimurium infection [16]. In Ity ${ }^{\mathrm{R}}$ mice, MФ express a functional Nramp1 gene, and removal of $M \Phi$ may therefore adversely effect the innate resistance of naive mice to virulent S. typhimurium infections. It is not known whether humans express alleles of Nramp1, but promoter polymorphisms, which may yield phenotypes similar to the murine Ity $^{S}$ and Ity $^{\mathrm{R}}$, have been observed [37]. However, further understanding of the mechanism behind M $\Phi$-mediated pathology of S. typhimurium infections in susceptible individuals would be of great benefit for the development of therapeutic strategies. Such new treatments, used in combination with antibiotic treatment, should focus on transient inhibition of early release of cytokines and/or other inflammatory mediators by $M \Phi$ which are mainly responsible for pathology associated with Salmonella infections, thereby limiting the damage in infected tissues and septicimia, and potentially reducing the clinical symptoms of typhoid fever.

\section{Materials and methods}

\subsection{Bacteria}

In this study we used the $\operatorname{aro} A$ aroD mutant of S. typhimurium SL1344 (BRD509, a kind gift of Prof. G. Dougan, Imperial College, London, GB) expressing C-frag from the pTETtac4 expression construct (BRD509/C-frag) [38], and virulent S. typhimurium SL1344 [39].

\subsection{In vivo $\mathrm{M \Phi}$ depletion}

$\mathrm{M} \Phi$ can be depleted in vivo using $\mathrm{Cl}_{2} \mathrm{MDP}$-loaded liposomes [22-25]. We have previously established that $\mathrm{Cl}_{2} \mathrm{MDP}$ does not affect the growth of BRD509 or SL1344 in vitro (results not shown). Liposomes were prepared as previously described [22]; $\mathrm{Cl}_{2} \mathrm{MDP}$ was a kind gift of Boehringer Mannheim (Mannheim, Germany). In some experiments, mice were treated with PBS-loaded liposomes as a control. Successful elimination of $\mathrm{M} \Phi$ from spleen and liver was confirmed by immunohistology.

\subsection{Immunization protocol}

BRD509, BRD509/C-frag and SL1344 were grown overnight in stationary Luria-Bertani (LB) broth cultures (supplemented with antibiotics) at $37^{\circ} \mathrm{C}$ and were diluted with PBS. Normal and $\mathrm{Cl}_{2} \mathrm{MDP}$-liposome-treated 6- to 8-week-old female BALB/c mice (lty ${ }^{\mathrm{s}}$ ) were inoculated i.v. with $10^{5} \mathrm{CFU}$ BRD509/C-frag or BRD509 in $0.2 \mathrm{ml}$ PBS. When indicated, mice were challenged i.v. with 100 CFU virulent SL1344 $\left(10 \times \mathrm{LD}_{50}\right)$ in $0.2 \mathrm{ml}$ PBS.

\subsection{Viable counts of S. typhimurium in organs}

Groups of five mice were killed at indicated time points after infection, and aseptically removed spleens and livers were homogenized in sterile bags (Starstedt, Ingle Farm, SA, Australia) containing $5 \mathrm{ml}$ of sterile PBS, using a Stomacher 80 homogenizer (Seward Medical, London, GB). The number of viable S. typhimurium in tissue homogenates was determined by viable count on LB agar plates containing the appropriate antibiotics.

\subsection{Detection of antigen-specific antibodies in serum by ELISA}

Serum antibodies against C-frag or LPS were detected by ELISA on tetanus toxin-coated ( $2 \mathrm{Lf} / \mathrm{ml}$, CSL Ltd, Melbourne, Austalia) or S. typhimurium LPS-coated $(10 \mu \mathrm{g} / \mathrm{ml}$, Sigma Chemical Co., MO) microtiter plates (NUNC, Denmark) respectively, as described in detail previously [40]. Serum titers are presented as the highest dilution with an absorbance of 0.10 above normal mouse serum.

\subsection{Lymphocyte proliferation assay}

$\mathrm{T}$ cell proliferation assays were performed 10 days after immunization in 96-well flat-bottom plates (NUNC, Denmark) in triplicate cultures. Single-cell suspensions prepared from spleens of three mice were enriched for T cells by passing through nylon wool columns. Splenic T cells $\left(3 \times 10^{5} /\right.$ well) were cultured with serial dilutions of recombinant $C$ frag and $3 \times 10^{5}$ irradiated (3000 rad) naive splenocytes as 
stimulators in RPMI 1640 (Gibco-BRL Laboratories, Grand Island, NY) supplemented with $10 \%$ FCS, 2 mM glutamine, antibiotics and 2-ME. The cells were cultured for $96 \mathrm{~h}$ and the last $16 \mathrm{~h}$ pulsed with $\left[{ }^{3} \mathrm{H}\right]$ thymidine. Cells harvested on fiberglass filters and $\left[{ }^{3} \mathrm{H}\right]$ thymidine incorporation was measured.

\subsection{Statistical analysis}

The non-parametric Mann-Whitney U-test was used to determine statistically significant differences between groups of mice.

\section{References}

1 Ivanoff, B., Levine, M. M. and Lambert, P. H., Vaccination against typhoid fever: present status. Bull. World. Health. Organ. 1994. 72: 957-971.

2 Carter, P. B. and Collins, F. M., The route of enteric infection in normal mice. J. Exp. Med. 1974. 139: 1189-1203.

3 Jensen, V. B., Harty, J. T. and Jones, B. D., Interactions of the invasive pathogens Salmonella typhimurium, Listeria monocytogenes, and Shigella flexneri with M cells and murine Peyer's patches. Infect. Immun. 1998. 66: 3758-3766.

4 Mastroeni, P., Villarreal-Ramos, B. and Hormaeche, C. E., Adoptive transfer of immunity to oral challenge with virulent salmonellae in innately susceptible BALB/c mice requires both immune serum and T-cells. Infect. Immun. 1993. 61: 3981-3984.

5 Hess, J., Ladel, C., Miko, D. and Kaufmann, S. H., S. typhimurium aro $A$-infection in gene-targeted immunodeficient mice: major role of $\mathrm{CD}^{+}{ }^{+} \mathrm{TCR}-\alpha \beta$ cells and IFN- $\gamma$ in bacterial clearance independent of intracellular location. J. Immunol. 1996. 156: 3321-3326.

6 Maskell, D. J., Hormaeche, C. E., Harrington, K. A., Joysey, H. S. and Liew, F. Y., The initial suppression of bacterial growth in a Salmonella infection is mediated by a localized rather than a systemic response. Microb. Pathog. 1987. 2: 295-305.

7 Hormaeche, C. E., The natural resistance of radiation chimeras to S. typhimurium C5. Immunology 1979. 37: 329-332.

8 Rathman, M., Barker, L. P. and Falkow, S., The unique trafficking pattern of $S$. thyphimurium-containing phagosomes in murine macrophages is independent of the mechanism of bacterial entry. Infect. Immun. 1997. 65: 1475-1485.

9 Alpuche Aranda, C. M., Swanson, J. A., Loomis, W. P. and Miller, S. I., S. thyphimurium activates virulence gene transcription within acidified macrophage phagosomes. Proc. Natl. Acad. Sci. USA 1992. 89: 10079-10083.

10 Buchmeier, N. A. and Heffron, E., Inhibition of macrophage phagosome-lysosome fusion by S. typhimurium. Infect. Immun. 1991. 59: 2232-2238.

11 Monack, D. M., Raupach, B., Hromockyj, A. E. and Falkow, S., S. typhimurium invasion induces apoptosis in infected macrophages. Proc. Natl. Acad. Sci. USA 1996. 93: 9833-9838.

12 Dunlap, N. E., Benjamin, W., Jr., Berry, A. K., Eldrige, J. H. and Briles, D. E., A 'safe-site' for S. typhimurium is within splenic polymorphonuclear cells. Microb. Pathog. 1992. 13: 181-190.

13 Conlan, J. W. and North, R. J., Early pathogenesis of infection in the liver with the facultative intracellular bacteria Listeria monocytogenes, Francisella tularensis, and Salmonella typhimurium involves lysis of infected hepatocytes by leukocytes. Infect. Immun. 1992. 60: 5164-5171.
14 Richter-Dahlfors, A., Buchan, A. and Finlay, B. B., Murine salmonellosis studied by confocal microscopy: S. typhimurium resides intracellularly inside macrophages and exerts a cytotoxic effect on phagocytes in vivo. J. Exp. Med. 1997. 186: 569-580.

15 Plant, J. and Glynn, A. A., Natural resistance to Salmonella infection, delayed hypersensitivity and Ir genes in different strains of mice. Nature 1974. 248: 345-347.

16 Vidal, S., Tremblay, M. L., Govoni, G., Gauthier, S., Sebastiani, G., Malo, D., Skamene, E., Olivier, M., Jothy, S. and Gros, P., The Ity/Lsh/Bcg locus: natural resistance to infection with intracellular parasites is abrogated by disruption of the Nramp1 gene. J. Exp. Med. 1995. 182: 655-666.

17 Vidal, S. M., Pinner, E., Lepage, P., Gauthier, S. and Gros, P., Natural resistanct to intracellular infections: Nramp1 encodes a membrane phosphoglycoprotein absent in macrophages from susceptible (Nramp1 D169) mouse strains. J. Immunol. 1996. 157: 3559-3568.

18 Mastroeni, P., Villarreal-Ramos, B. and Hormaeche, C. E., Role of T-cells, TNF $\alpha$ and IFN- $\gamma$ in recall of immunity to oral challenge with virulent salmonellae in mice vaccinated with live attenuated aro-Salmonella vaccines. Microb. Pathog. 1992. 13: 477.

19 Harding, C. V. and Pfeiffer, J. D., Antigen expressed by S. typhimurium is processed for class I major histocomptibility complex presentation by macrophages but not infected epithelial cells. Immunology 1994. 83: 670-674.

20 Verjans, G. M., Janssen, R., UytdeHaag, F. G., van Doornik, C. E. and Tommassen, J., Intracellular processing and presentation of T-cell epitopes, expressed by recombinant Escherichia coli and S. typhimurium, to human T cells. Eur. J. Immunol. 1995. 25: 405-410.

21 Banchereau, J. and Steinman, R. M., Dendritic cells and the control of immunity. Nature 1998. 392: 245-252.

22 Van Rooijen, N. and Sanders, A., Liposome mediated depletion of macrophages: mechanism of action, preparation of liposomes and applications. J. Immunol. Methods 1994. 174: 83-93.

23 Nair, S., Buiting, A. M., Rouse, R. J., Van Rooijen, N., Huang, L. and Rouse, B. T., Role of macrophages and dendritic cells in primary cytotoxic T lymphocyte responses. Int. Immunol. 1995. 7: 679-688.

24 Ciavarra, R. P., Buhrer, K., Van Rooijen, N. and Tedeschi, B., Tcell priming against vesicular stomatitis virus analyzed in situ: red pulp macrophages, but neither marginal metallophilic nor marginal zone macrophages, are required for priming CD4 ${ }^{+}$and CD8 ${ }^{+}$ T-cells. J. Immunol. 1997. 158: 1749-1755.

25 Qian, Q., Jutila, M. A., Van Rooijen, N. and Cutler, J. E., Elimination of mouse splenic macrophages correlates with increased susceptibility to experimental disseminated candidiasis. J. Immunol. 1994. 152: 5000-5008.

26 Fraser, C. C., Chen, B. P., Webb, S., van Rooijen, N. and Kraal, G., Circulation of human hematopoietic cells in severe combined immunodeficient mice after $\mathrm{Cl}_{2} \mathrm{MDP}$-liposome-mediated macrophage depletion. Blood 1995. 86: 183-192.

27 O'Brien, A. D., Scher, I. and Formal, S. B., Effect of silica on the innate resistance of inbred mice to S.typhimurium infection. Infect. Immun. 1979. 25: 513-520.

28 Gulig, P. A., Doyle, T. J., Hughes, J. A. and Matsui, H., Analysis of host cells associated with the Spv-mediated increased intracellular growth rate of S. typhimurium in mice. Infect. Immun. 1998. 66: 2471-2385.

29 Khan, S. A., Everest, P., Servos, S., Foxwell, N., Zahringer, U., Brade, H., Rietschel, E. T., Dougan, G., Charles, I. G. and Maskell, D. J., A lethal role for lipid A in Salmonella infections. Mol. Microbiol. 1998. 29: 571-579.

30 Salkowski, C. A., Neta, R., Wynn, T. A., Strassmann, G., van Rooijen, N. and Vogel, S. N., Effect of liposome-mediated macrophage depletion on LPS-induced cytokine gene expression and radioprotection. J. Immunol. 1995. 155: 3168-3179. 
31 Eisenstein, T. K., Deakins, L. W., Killar, L., Saluk, P. H. and Sultzer, B. M., Dissociation of innate susceptibility to Salmonella infection and endotoxin responsiveness in $\mathrm{C} 3 \mathrm{HeB} / \mathrm{FeJ}$ mice and other strains in the $\mathrm{C} 3 \mathrm{H}$ lineage. Infect. Immun. 1982. 36: 696-703.

32 Soo, S. S., Villarreal-Ramos, B., Anjam Khan, C. M., Hormaeche, C. E. and Blackwell, J. M., Genetic control of immune response to recombinant antigens carried by an attenuated S. typhimurium vaccine strain: Nramp1 influence T-helper subset responses and protection against leishmanial challenge. Infect. Immun. 1998. 66: 1910-1917.

33 Lang, T., Prina, E., Sibthorpe, D. and Blackwell, J. M., Nramp1 transfection transfers Ity/Lsh/Bcg-related pleiotropic effects on macrophage activation: influence on antigen processing and presentation. Infect. Immun. 1997. 65: 380-386.

34 Marriott, I., Hammond, T. G., Thomas, E. K. and Bost, K. L., Salmonella efficiently enter and survive within cultured CD11 ${ }^{+}$ dendritic cells initiating cytokine expression. Eur. J. Immunol. 1999. 29: 1107-1115.

35 Hopkins, S. A. and Kraehenbuhl, J. P., Dendritic cells of the murine Peyer's patches colocalize with S. typhimurium avirulent mutants in the subepithelial dome. Adv. Exp. Med. Biol. 1997. 417: 105-109.

36 de Jong, R., Altare, F., Haagen, I. A. et al., Severe mycobacterial and Salmonella infections in interleukin-12 receptor-deficient patients. Science 1998. 280: 1435-1438.
37 Blackwell, J. M., Barton, C. H., White, J. K., Searle, S., Baker, A. M., Williams, H. and Shaw, M. A., Genomic organization and sequence of the human NRAMP gene: identification and mapping of a promoter region polymorphism. Mol. Med. 1995. 1: 194.

38 Fairweather, N. F., Chatfield, S. N., Makoff, A. J., Strugnell, R. A., Bester, J., Maskell, D. J. and Dougan, G., Oral vaccination of mice against tetanus by use of a live attenuated Salmonella carrier. Infect. Immun. 1990. 58: 1323-1326.

39 Hoiseth, S. K. and Stocker, B. A., Aromatic-dependent S. typhimurium are non-virulent and effective as live vaccines. Nature 1981. 291: 238-239.

40 Wijburg, O. L. C., Van den Dobbelsteen, G. P., Vadolas, J., Sanders, A., Strugnell, R. A. and Van Rooijen, N., The role of macrophages in the induction and regulation of immunity elicited by exogenous antigens. Eur. J. Immunol. 1998. 28: 479-487.

Correspondence: Odilia L. C. Wijburg, Dept. of Microbiology and Immunology, The University of Melbourne, Parkville 3052, Melbourne, Australia

Fax: +61-3-93471540

e-mail: o.wijburg@microbiology.unimelb.edu.au 